\title{
The impact of a specialized spinal cord injury center as compared with non-specialized centers on the acute respiratory management of patients with complete tetraplegia: an observational study
}

\author{
Andréane Richard-Denis ${ }^{1,2} \cdot$ Debbie Feldman $^{2} \cdot$ Cynthia Thompson $^{1} \cdot$ Martin Albert $^{1,2} \cdot$ Jean-Marc Mac-Thiong $^{1,2,3}$
}

Received: 15 June 2017 / Revised: 12 August 2017 / Accepted: 14 August 2017 / Published online: 15 November 2017

(C) International Spinal Cord Society 2018

\begin{abstract}
Study Design Retrospective cohort study.

Objectives To compare the proportion of tracheostomy placement and duration of mechanical ventilation (MV) in patients with a complete cervical spinal cord injury (SCI) that were managed early or lately in a specialized acute SCI-center. The second objective was to determine the impact of the timing of admission to the SCI-center on the MV support duration.
\end{abstract}

Setting A single Level-1 trauma center specialized in SCI care in Quebec (Canada).

Methods A cohort of 81 individuals with complete tetraplegia over a 6-years period was included. Group $1(N=57$ - early group-) was admitted before surgical management in one specialized acute SCI-center, whereas Group 2 ( $N=24$-late group-) was surgically managed in a non-specialized center and transferred to the SCI-center for post-operative management only. The proportion of tracheostomy placement and MV duration were compared. Multivariate regression analysis was used to assess the impact of the timing of admission to the SCI-center on the MV duration during the SCI-center stay.

Results Patients in Group 2 had a higher proportion of tracheostomy (70.8 vs. 35.1\%, $p=0.004)$ and a higher mean duration of MV support $(68.0 \pm 64.2$ days vs. $21.8 \pm 29.7$ days, $p=0.006)$ despite similar age, trauma severity (ISS), neurological level of injury and proportion of pneumonia. Later transfer to the specialized acute SCI-center was the main predictive factor of longer MV duration, with a strong impact factor $(s=946.7, p<0.001)$.

Conclusions Early admission to a specialized acute SCI-center for surgical and peri-operative management after a complete tetraplegia is associated with lower occurrence of tracheostomy and shorter mechanical ventilation duration support.

Sponsorship MENTOR Program of the Canadian Institute of Health Research and US Department of Defense Spinal Cord Injury Research Program.

\section{Introduction}

Acute cervical spinal cord injury (SCI) is a devastating condition for the respiratory system and a leading cause of

Andréane Richard-Denis

andreane.rdenis@gmail.com

1 Research Center, Hopital du Sacré-Cœur de Montréal, Montréal, QC, Canada

2 Faculty of Medicine, University of Montreal, CP 6128, Succ Centre-ville, Pavillon 7077 Avenue du Parc, Montréal, Québec, Canada

3 CHU Ste-Justine, Montreal, Canada morbidity and mortality [1, 2]. The proportion of cervical spine trauma has increased in the last years in Canada, reaching $60 \%$ of all traumatic SCI [3, 4]. The level and completeness of the SCI are major determinants of the severity of the respiratory condition; the more rostral and complete the injury, the greater the likelihood of respiratory impairment [1, 2].

Individuals with cervical SCI exhibit reduced lung volumes and flow rates as a result of respiratory muscle dysfunction $[1,5]$. They experience decreased lung expansion, highly impaired cough due to weakness of the expiratory muscles, and increased sputum production due to 
unopposed sympathetic stimulation and decreased surfactant production [2]. Individuals with acute complete tetraplegia are particularly vulnerable to respiratory failure during the acute phase resulting from the combined effect of spinal shock leading to flaccid chest wall muscles, denervation of the ventilatory muscles, presence of concomitant lung injuries, potential decreased respiratory drive due to concomitant head injuries and narcotic analgesic, and cervical soft tissue edema associated with the surgery [5]. Between 40 and $80 \%$ of subjects with acute complete tetraplegia may therefore require mechanical ventilation (MV) support during the acute care [6-8]. As prolonged oral/nasal endotracheal tubes is detrimental, tracheostomy placement is required in $10-60 \%$ of patients following tetraplegia $[6,9,10]$.

Important predictors of MV support requirement following acute SCI include the level and completeness of the SCI, followed by lack of diaphragm function, advanced age, and pneumonia $[6,7,10]$. With regards to pulmonary function, spontaneous improvement in the vital capacity (VC) and the first expiratory volume in the first second (FEV1) were also important predictors of MV support [7]. Not only is requirement for MV support associated with the occurrence of pneumonia (ventilator-associated pneumonia) [7] and decreased quality of life [11], it is also one of the costliest consequences of tetraplegia, as it is associated with longer intensive care unit (ICU) stay, higher hospital costs, significant infection risks, as well as social isolation and decreased functional outcome $[8,12]$. Requirement for MV is also an important risk factor of pneumonia and individuals sustaining complete tetraplegia, even at higher levels of injury, may be successfully weaned from MV during acute care $[6,8,12]$. Thus, it is important to attempt weaning in a timely and appropriate fashion to potentially improve respiratory and functional outcome, as well as survival rate $[7,8]$.

Individuals sustaining an acute traumatic SCI are generally first transferred to the nearest hospital center for evaluation and medical stabilization. After stabilization, the regional medical team has to decide whether a prompt surgery at the non-specialized regional center or direct transfer to the SCI-center should be prioritized. Specialized acute SCI-centers are familiar with the respiratory management of individuals with cervical SCI (based on the evidence-based) [2, 13-16], resulting in decreased occurrence of respiratory complications and increased rate of successful weaning of MV support [2, 7, 12]. A recent systematic review has also suggested that early transfer (within $48 \mathrm{~h}$ ) to a specialized acute SCI-center may decrease as much as $50 \%$ the total length of stay and decrease the rate and severity of complications, as well as decrease mortality [14]. However, even if early transfer to the specialized acute SCI-center is recommended, it remains a low-level recommendation (level V-expert opinion) [13]. On the other hand, recent studies have suggested that emergent spinal surgery could improve neurological recovery, decrease costs of care and the incidence of complications following traumatic SCI [14, 17-20]. So, optimal timing for transfer to SCI-center should be established with respect to the spinal surgical procedure and the amount of specialized perioperative care provided. This is particularly important for complete cervical SCI, as this condition is associated with limited neurological recovery and a high risk of complications. The impact of transfer delays on relevant respiratory clinical features remains also unknown. On the basis of the conclusions of several important studies [8, 12, 14, 21], we hypothesized that individuals with complete tetraplegia managed early before surgery in a SCI-center will be less likely to require tracheostomy placement and would be weaned faster from MV support than patients undergoing surgical management in a non-specialized (NS) center with subsequent transfer to a SCI-center. Accordingly, the objectives of this study were to (1) compare the proportion of tracheostomy placement and MV duration in patients with a complete cervical traumatic SCI admitted to a specialized acute SCI-center before surgery or only after surgical management in a non-specialized center, and (2) determine the impact of the timing of admission to the SCI-center on MV support duration using a multivariate regression analysis.

\section{Methods}

\section{Patients}

Individuals sustaining a complete SCI (between $\mathrm{C} 1$ and C8) were selected from a prospective database of individuals admitted to a single Level I specialized acute SCI-center between April 2008 and November 2014. This database has already been partially used for other studies [21, 22]. As we receive around 70 patients from the Western par of the province of Quebec with a traumatic SCI per year in our center, the recruitment of patients for this study was relatively stable over the years. Thus, 81 patients (63 males/18 females; $43.0 \pm 18.0$ years old) were included in this study. A complete injury consisted in a "grade A" injury on the American Spinal Injury Association impairment scale (AIS), according to the International Standards for Neurological Classification of Spinal Cord Injury (ISNCSCI). All patients were treated surgically to decompress and stabilize the spine. Subjects managed non-surgically or with an incomplete cervical SCI were excluded as they exhibit distinct outcomes.

In line with the objectives of this study, our cohort was subdivided into two groups based on the timing of 
admission to the specialized center. No patients sustaining an acute complete tetraplegia were completely managed by a non-specialized center before transfer to the intensive functional rehabilitation, without prior admission to the specialized acute SCI-center. In other words, all patients with a complete traumatic cervical SCI that occurred in the western part of Quebec were at some point managed in our specialized acute SCI-center during the period of recruitment of this study. Group 1 included 57 individuals transferred "early" to the specialized acute SCI-center, where "early" was defined as an admission to a single acute Level-I specialized acute SCI-center before surgical management to receive complete peri-operative management by a multidisciplinary team specialized in the acute care and acute rehabilitation of 80-100 SCI patients per year. Group 2 included 24 patients transferred "lately" to the specialized acute SCI-center for late postoperative management only after surgical management in a non-specialized center. A total of four patients (7\%) from Group 1 have been transported from the trauma site to a non-specialized center for initial evaluation, but were transferred to the specialized acute SCI-center when a SCI was suspected, in order to be surgically managed at the SCI-center.

The organization of SCI care can vary significantly between different regions. In Quebec, Canada, patients sustaining a traumatic SCI should be directed to one of two designated specialized acute SCI-centers according to the geographical region where the trauma occurred (eastern vs. western part of the province). This system was established in the late 1970s to allow centralization of patients and improve the care of SCI patients, based on the general principles originally devised in the United States [23].

The specialized acute SCI-center involved in the current study offers specialized respiratory management administered by a multidisciplinary team specialized in SCI care. It is the specialized acute care center for ventilation-assisted patients designated for the province of Quebec. This team is composed of, but not limited to, trauma, intensive care, spine surgery and physical medicine, and rehabilitation specialists, as well as many therapists and clinical nurses experienced in SCI care. All patients with complete tetraplegia are admitted to the ICU and transferred to the ward once their condition is considered stable by the intensive care team. All patients, whether admitted before (Group 1) or after spinal surgery (Group 2), undergo the same multidisciplinary management. Respiratory care is individualized for each patient and indications for endotracheal intubation, tracheostomy or MV support follow evidence-based recommendations for the acute care of SCI $[2,13,15,16]$. Routine respiratory care for complete cervical SCI involves high frequency percussive ventilation, mechanical and/or manual assisted cough, and non-invasive respiratory assistance or MV support if needed $[2,13,15,16]$. Readiness for weaning of MV support is challenged, and managed by the intensive care medical team, when vital capacity reaches at least $15 \mathrm{mg} / \mathrm{kg}$ along with decreased sputum load, ability to cooperate, patent upper airway, relatively clear chest radiograph with no new changes, and reduction in the requirement for ventilator assistance [9]. A progressive ventilator-free breathing protocol with high tidal volume is preconized [2]. Tracheostomy tube is considered in patients that are anticipated to require ventilator support for more than two weeks [24]. Specialized rehabilitation therapies are provided continuously throughout hospitalization and include respiratory, physical and speech therapies, and nutrition services. A physical medicine and rehabilitation specialist leads the acute rehabilitation process, applying interventions to promote functional and neurological recovery and prevent medical complications. The physical medicine and rehabilitation specialist also coordinates transfer to a functional rehabilitation facility once the patient's condition does not require additional active medical or surgical intervention.

\section{Data collection}

The local ethics committee board approved this study. Data pertaining to the hospitalization at the SCI-center were prospectively collected by independent research assistants and a medical archivist. For patients in Group 2, chart review was required to collect information pertaining to the presence of complications upon admission to the specialized acute SCI-center.

The following variables were collected in the medical chart: age, gender, and history of chronic obstructive pulmonary disease (COPD) (chronic bronchitis, emphysema, asthma, and bronchiolitis-confirmed in the chart by a health-care professional-). The weight and height (to determine the presence of obesity (defined with a body mass index of $\geq 30$ )) and history of smoking (current, former, or non-smoker) were asked to the patient on admission. Clinical data, including the trauma severity (as measured by the Injury Severity Score (ISS)), presence of a concomitant traumatic brain injury (TBI) and its severity (mild, moderate, or severe), surgical delay, and information regarding the SCI, were also collected. The ISNCSCI were used to assess the severity of neurological injury through the AIS grade within the first $72 \mathrm{~h}$ following the SCI for Group 1, and upon admission for Group 2 [25]. The neurological level of injury (NLI) was defined as the most caudal segment with normal motor and sensory function bilaterally, as assessed by a trained medical team member within the first $72 \mathrm{~h}$ following the SCI for Group 1, and upon admission for Group 2. The NLI was dichotomized as high tetraplegia 
Table 1 Socio-demographic and clinical characteristics of patients with a complete cervical SCI based on the timing of admission to the SCI-center $(n=81)$

\begin{tabular}{|c|c|c|c|c|c|}
\hline \multirow[t]{2}{*}{ Characteristics } & & & \multicolumn{2}{|l|}{ Admission to the SCI-center } & \multirow[t]{2}{*}{$P$-value } \\
\hline & & & \multirow{2}{*}{$\begin{array}{l}\text { Before surgery (Group 1) } \\
57\end{array}$} & \multirow{2}{*}{$\begin{array}{l}\text { After surgery (Group 2) } \\
24\end{array}$} & \\
\hline$N$ & & & & & - \\
\hline \multirow{2}{*}{ Age (years) } & Median (IQR) & & $41.0(29.3-57.0)$ & $45.5(22.0-55.0)$ & 0.83 \\
\hline & Mean \pm SD & & $43.6 \pm 17.8$ & $42.5 \pm 19.0$ & \\
\hline Gender & $\%$ Male & & 75.4 & 83.3 & 0.56 \\
\hline \multirow[t]{3}{*}{ Smoking status } & $\%$ Non-smoker & & 56.1 & 62.5 & 0.31 \\
\hline & $\%$ Active smoker & & 22.8 & 29.2 & \\
\hline & $\%$ Former smoker & & 21.1 & 8.3 & \\
\hline Obesity & $\%$ Body mass index $\geq 30$ & & 1.8 & 4.2 & 0.51 \\
\hline COPD & $\%$ COPD & & 0 & 0 & 1.00 \\
\hline \multirow[t]{2}{*}{ ISS } & Median (IQR) & & $29.0(25.0-42.0)$ & $34.0(25.0-71.0)$ & 0.31 \\
\hline & Mean \pm SD & & $35.3 \pm 16.0$ & $42.7 \pm 20.9$ & \\
\hline NLI & $\% \mathrm{C} 1-\mathrm{C} 4$ & & 56.1 & 66.7 & 0.46 \\
\hline \multirow[t]{4}{*}{ TBI } & $\%$ None & & 49.1 & 75.0 & 0.06 \\
\hline & $\%$ Mild & & 43.9 & 20.8 & \\
\hline & $\%$ Moderate & & 3.5 & 4.2 & \\
\hline & $\%$ Severe & & 3.5 & 0.0 & \\
\hline Pneumonia & $\%$ Pneumonia & & 50.9 & 41.7 & 0.48 \\
\hline \multirow[t]{3}{*}{ Surgical delay $(\mathrm{h})$} & $\% \leq 24 \mathrm{~h}$ post-trauma & & 54.4 & 29.2 & 0.051 \\
\hline & Median (IQR) & & $23.0(14.9-37.3)$ & $23.0(14.9-36.2)$ & 0.50 \\
\hline & Mean \pm SD & & $54.9 \pm 1296$ & $80.3 \pm 1971$ & \\
\hline In-hospital death & $\%$ Deceased & & 8.8 & 8.3 & 1.00 \\
\hline \multirow[t]{6}{*}{ Length of stay (days) } & SCI-center(Median (IQR)Mean \pm SD) & ICU & $17.0(8.5-41.5)$ & $35.5(8.0-91.5)$ & 0.13 \\
\hline & & & $28.1 \pm 28.0$ & $49.2 \pm 44.8$ & \\
\hline & & Total & $48.0(24.0-72.5)$ & $68.5(32.5-120.0)$ & 0.02 \\
\hline & & & $57.0 \pm 43.3$ & $78.0 \pm 45.9$ & \\
\hline & \multirow{2}{*}{\multicolumn{2}{|c|}{$\begin{array}{l}\text { Total acute care: non-specialized center }+ \text { SCI-center } \\
\text { (Median (IQR) Mean } \pm \text { SD) }\end{array}$}} & $49.0(24.5-72.5)$ & $95.5(57.8-146.8)$ & $<0.001$ \\
\hline & & & $57.0 \pm 43.3$ & $105.3 \pm 56.5$ & \\
\hline
\end{tabular}

$S C I$ Spinal Cord injury, COPD chronic obstructive pulmonary disease, ISS Injury Severity Score, $N L I$ neurological level of injury, TBI traumatic brain injury, ICU intensive care unit, $N S$-center non-specialized, $I Q R$ Interquartile range. Bold values signify that $P$-value is significant $<0.05$.

(C1-C4), and low tetraplegia (C5-C8). The surgical delay was defined as the number of hours between the trauma and the timing of surgical incision and dichotomized with a cut-off of $24 \mathrm{~h}$ based on previous studies [26-28]. The occurrence of pneumonia during the hospitalization in the SCI-center was collected. Pneumonia was defined as a new progressing lung infiltrate accompanied by at least 2 of the following: (1) body temperature higher than $38^{\circ}$ or lower than $36^{\circ}$ Celsius; (2) leukocytosis greater than 12,000 or leukopenia below 4000 per $\mathrm{ml}$; (3) purulent pulmonary secretions [15]. The surgical delay was defined as the time (in hours) between the trauma and spinal surgery (time of skin incision), and was dichotomized as $<24$ or $\geq 24 \mathrm{~h}$ post-trauma. The acute care length of stay, both in the ICU and the ward, as well as the rate of mortality were noted.

\section{Outcome variables}

The main outcome variable was the percentage of individuals who required tracheostomy and MV support as well as the ventilation support duration (in days) at the SCIcenter. The proportion of individuals requiring tracheostomy placement, whether the procedure was performed at the specialized acute SCI-center or the non-specialized facility (Group 2) was also calculated, in addition to the proportion of patients requiring MV support. The MV duration was defined as the total number of hours for which patients required MV support during their stay at the specialized acute SCI-center. If intermittent episodes of ventilation were necessary, the number of hours for each of the episodes was added. The MV duration time was then translated into days for both groups. 
Table 2 Respiratory outcomes in patients with a complete cervical SCI early and lately admitted to the SCI-center $(n=81)$

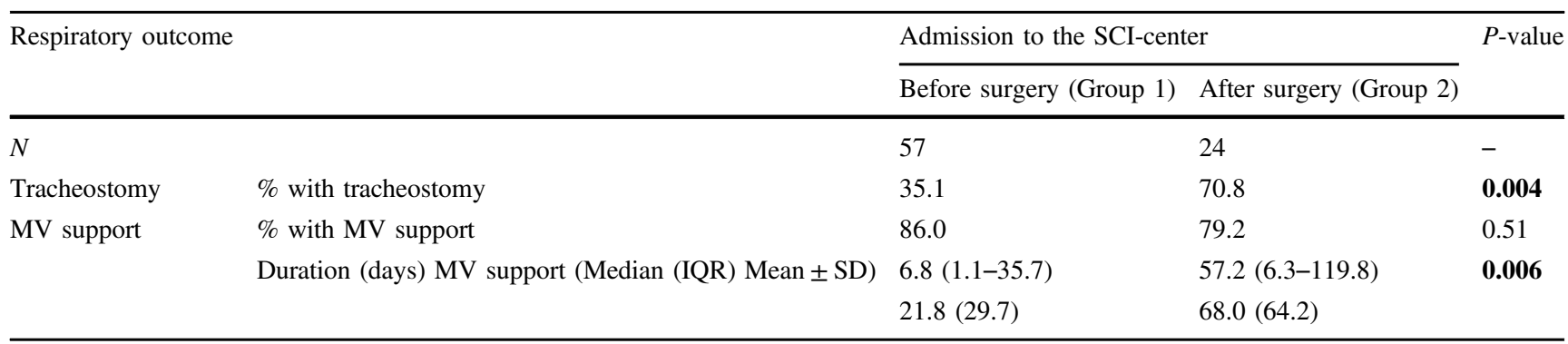

$S D$ standard deviation, $M V$ mechanical ventilation. Bold values signify that $P$-value is significant $<0.05$.

\section{Statistical analyses}

Direct comparison analyses were first performed to assess the first objective of this study. Continuous data were compared between the two groups using Mann-Whitney U tests, while categorical data were compared using chisquare tests. Continuous data were reported as median and interquartile range (IQR), and categorical data were reported as proportions and percentages.

Then, the second objective of this study was assessed using a multivariate linear regression analysis based on a general linear model in order to identify predictors of MV support duration and evaluate the impact of timing of admission to the SCI-center (Group 1 or 2). As a first step, multicollinearity between potential factors was tested using Spearman correlations, with a cut-off value of 0.7 . Since no collinearity was found between the independent variables, they were all included in the general linear model. The dependent variable was the MV support duration (in days) during the SCI-center stay, and ten independent variables were included: (1) timing of admission to the SCIcenter (main independent variable); (2) age; (3) gender; (4) smoking status (non-smoker vs. former or active smoker); (5) the presence of obesity; (6) ISS; (7) occurrence of pneumonia; (8) high (C1-4) or low (C5-8) tetraplegia; (9) the presence of concomitant TBI, and (10) surgical delay $(<24$ vs. $\geq 24 \mathrm{~h}$ ). We have also performed the same general linear model with the surgical delay as a continuous variable (instead of dichotomous). We used a backward elimination method, and the magnitude of the impact of each significant factor was reported using the beta coefficient. The R-square refers to the percentage of variance of the dependent variable explained by the final model. The significance level was set at $p<0.05$ and all statistical analyses were performed using the IBM SPSS Statistics 19 software (Chicago, IL).

\section{Results}

A total of 81 subjects with acute traumatic complete tetraplegia were included in this study. Group 1 included 57 patients admitted early to the specialized acute SCI-center (Group 1), while 24 subjects were transferred later to the specialized acute SCI-center (Group 2). No patients sustaining a complete traumatic cervical SCI were treated conservatively (non-surgically) during the period of recruitment. Baseline characteristics, occurrence of pneumonia during acute care and mortality rate exhibited nonsignificant differences between the two groups (Table 1). The proportion of patients sustaining concomitant mild TBI tended to be greater for Group $1(p=0.06)$, but the difference was not statistically significant. Patients in Group 1 were more likely to have surgery within $24 \mathrm{~h}(p=0.051)$. Indeed, the mean surgical delay for Group 1 reached 55 days as compared with 80 days for Group 2. However, this difference was not significant. The median surgical delay was similar between the two groups ( 23 days).

Patients from Group 2 spent on average $27.2 \pm 28.4$ days in the non-specialized hospital before their transfer to the SCI-center. While the majority of Group 1 patients (53/57) were initially evaluated in a community hospital before their transfer to the SCI-center, the mean delay between trauma and admission at the specialized acute SCI-center was about $7 \mathrm{~h}(0.3 \pm 1.0$ day $)$. The total length of stay in the specialized acute SCI-center was significantly increased by 20 days in Group $2(p<0.001)$. The length of stay in the ICU at the SCI-center was increased by 18 days in Group 2, but this difference did not reach statistical significance $(p=0.13)$ (Table 1).

The comparison of respiratory outcomes for Groups 1 and 2 is shown in Table 2. The proportion of patients requiring tracheostomy placement was doubled in Group 2 ( $p=0.004)$. Although the proportion of patients requiring MV support was similar in both groups, the duration of MV was increased by 46 days for patients in Group 2.

Our results from the multivariate analysis are shown in Table 3. The timing of admission to the specialized acute SCI-center was the most important predictor of the duration 
Table 3 Predictors of duration of mechanical ventilation (in days) for subjects with a complete cervical SCI: results of the multivariate analysis $(n=81)$

\begin{tabular}{llll}
\hline Predictors & & Beta coefficient (95\% CI) & $P$-value \\
\hline Timing of admission at SCI-center & Before surgery (Group 1) & $-946.7(-1413.6,-479.7)$ & $<0.001$ \\
& After surgery (Group 2) & Reference category & \\
NLI & C1-C4 & $588.7(142.2,1035.2)$ & 0.010 \\
& C5-C8 & Reference category & 0.036 \\
\hline
\end{tabular}

$N L I$ Neurological level of injury, $C I$ confidence interval, ISS injury severity score. Bold values signify that $P$-value is significant $<0.05$.

of MV, as patients transferred to the specialized acute SCIcenter only after surgery (Group 2) required longer MV. Higher NLI (C1-C4) and an increased ISS were significantly associated with increased MV duration. The final model explained almost $34 \%$ of the variance in MV support duration $\left(R^{2}=0.338\right)$. When the surgical delay was considered as a continuous variable (as opposed to a dichotomous variable), a longer surgical delay became a significant variable associated with longer duration of mechanical ventilation, with an impact factor of almost three. The presence of pneumonia during acute care was also added as a significant factor of longer mechanical ventilation duration when the surgical delay is treated as a continuous variable, with an important impact factor of 536.

\section{Discussion}

This comparative study suggests that following complete cervical SCI, early admission to a specialized acute SCIcenter for surgical and perioperative management (Group 1) decreases the duration of MV support and the rate of tracheostomy placement. The decreased duration in MV support gained with early admission to the specialized acute SCI-center reach numbers previously reported in the SCI literature (for complete tetraplegia) [29] and is even underestimated, considering that for patients transferred lately (Group 2) the duration of MV support in the nonspecialized center was not included despite a mean of 27.2 days spent in the non-specialized center before transfer in the specialized acute SCI-center. The benefits in respiratory care with early admission to a specialized acute SCI-center might be important as they are associated with a shorter length of stay for the acute care, and thereby earlier transfer to intensive functional rehabilitation. Secondarily, it is expected that complete surgical and perioperative management in a specialized acute SCI-center will decrease costs and resources use for this patient population for whom the economic burden is already significant [30].

Non-specialized centers may argue that performing surgery in their center will decrease the surgical delay, considering that a shorter surgical delay is associated with improved neurological recovery [31] and decreased incidence of complications [18], particularly for cervical SCI [19]. However in the current study, the surgical timing was similar when the surgery was performed in the specialized acute SCI-center (Group 1). This observation highlights the fact that transfer to the specialized acute SCI-center do not delay surgical management. It may also underline the ability of a specialized multidisciplinary team to organize prompt evaluation, medical stabilization and surgical planning, while there are multiple barriers to early surgery [26, 32]. However, the argument for earlier surgery in a nonspecialized center could still be considered in certain occasions for geographical reasons, and the influence of geographical distance on the need of early transfer to a SCI center should therefore be explored in a future study. This issue was not a factor in our study since the great majority of individuals from Group 2 had surgery in a non-specialized center located $<10 \mathrm{~km}$ away from our SCI-center.

MV support is generally required during the first days to weeks after the injury [2, 23, 33]. But as the chest wall muscle flaccidity transitions to spasticity and accessory muscles strengthen, spontaneous ventilation may be adequate for weaning from MV [2, 8, 33, 34]. The higher rate of tracheostomy in individuals lately transferred to the specialized acute SCI-center (Group 2) may reflect a false assumption that individuals with complete tetraplegia requiring MV support in the early-acute phase will remain ventilator-dependent. Alternatively, it can also suggest that weaning of $\mathrm{MV}$ in patients after a complete cervical SCI remains a difficult task if the treatment team in nonspecialized centers only deal with a small number of SCI patients in their usual practice and/or have not gained a wide experience with treating SCI patients during their training. Accordingly, Wong et al. have demonstrated that weaning attempts in individuals with complete tetraplegia acutely hospitalized in non-experienced centers were delayed or not attempted at all [2]. Yet, up to $60 \%$ of individuals with complete tetraplegia can be successfully weaned from MV during acute care [34]. Early admission to the specialized acute SCI-center, before surgery, may promote faster stabilization of the pulmonary status and more 
efficient weaning of MV, while decreasing the need for tracheostomy placement. These findings are further reinforced by the fact that a similar rate of mortality and incidence for MV support was found, suggesting that both groups were similarly vulnerable to respiratory failure.

As tracheostomy (as opposed to spontaneous ventilation), and MV support are recognized risk factors of pneumonia [24, 33-35], a higher frequency of pneumonia in patients with later referral (Group 2) was expected. Surprisingly, we found a similar frequency of pneumonia between individuals admitted earlier or later to the specialized acute SCI-center. Because the information pertaining to presence of pneumonia before the admission was not unavailable for this study, it is possible that the true incidence of pneumonia in Group 2 patients was underestimated, since these patients were transferred after a mean of 27.2 days after the SCI. Accordingly, it has been shown that pneumonia tends to develop early after a complete cervical SCI [36]. One may thus question if this may explain the significant higher rate of tracheostomy found in this group. Although it is not excluded, the fact that the same proportion of individuals required MV support does not support this hypothesis. If the occurrence of pneumonia in the non-specialized center had caused the higher rate of tracheostomy at arrival to the specialized acute SCI-center, we would have expected a higher rate of MV requirement as well, which was not the case in this study. Indeed, it rather suggests that individuals from Group 2 were transferred to the specialized acute SCI-center with a tracheostomy without requirement for MV support. This further supports that both groups were similarly vulnerable in terms of respiratory outcome, which was the main outcome of this study.

Our multivariate regression analysis further reinforces the importance of early transfer for optimizing the respiratory management in complete cervical SCI. Indeed, admission to the specialized acute SCI-center before surgical management was the strongest factor associated with shorter MV duration. It is interesting to note that when the surgical delay was considered as a continuous variable, longer surgical delays were also significantly associated with longer duration of MV. Considering that individuals lately transferred to the specialized acute SCI-center tended to be surgically-managed later (with a non-significant mean difference of 25 days), this result may reinforce the importance of early transfer regardless of the location of the injury. The level of injury was associated with MV duration, which is in accordance with the SCI literature [6, 7]. Indeed, complete injuries above $\mathrm{C} 4$ produce a significant ventilator muscle paralysis since the phrenic nerve, innervating the diaphragm, arises from the third to the fifth cervical roots [2]. Important breathing accessory muscles are also denervated, and the loss of expiratory muscle strength results in a severe cough impairment. As a result, these patients exhibit acute respiratory failure and typically do not survive unless MV support is rapidly instituted [13, 15, 16, 33]. Transition of the flaccid chest wall to spasticity and recruitment of accessory muscles required to initiate MV weaning may take a few weeks after injury $[2,16]$. Finally, the burden of associated traumatic injuries - assessed through the ISS in our study — was the last factor associated with MV duration during the SCI-center stay. Not only severe TBI may impair the respiratory drive, but also concomitant chest or lung injuries (such as pneumothorax, hemothorax, pulmonary contusions or flail chest) may further affect the pulmonary physiologic changes $[6,16,33]$.

Results of this study may suggest that both groups were similar in terms of severity and vulnerability to respiratory failure. Characteristics related to the patient (age, smoking status, presence of obesity (which may accentuate the restrictive lung syndrome), presence of COPD) and trauma (burden of associated traumatic injuries (as calculated by the ISS score), level of the SCI) were similar for both groups (Table 1). The severity of the SCI was also similar between the two groups since only individuals with complete SCI were included (AIS-A). There was a strong tendency towards a higher proportion of individuals with a concomitant traumatic brain injury in Group 1 (managed in the acute specialized SCI-center), which reinforces results of this study since an additional neurological injury may worsen outcome following SCI [37]. The fact that the proportion of individuals requiring mechanical ventilation support was similar further supports that both groups were similar in terms of severity. We therefore may suggest that difference of severity between the two groups is less likely to have influenced results of this study.

In conclusion, this study is in accordance with previous studies supporting the role of specialized SCI centers in decreasing the mortality and morbidity after a traumatic SCI $[2,12,15]$. However, our study is highly relevant because it specifically assesses the importance of a specialized acute SCI-center for improving the respiratory care in patients with a traumatic complete SCI. The healthcare system in Quebec comprises two specialized acute SCI-center in which all traumatic SCI should be transfer during the acute care management. Our center is also the designated acute care center for ventilated-assisted patients of the province of Quebec. Whether a patient should be transferred prior or after the spinal surgical procedure remains arbitrary, although this information is critical since it may influence the outcome of patients. Based on the results of this study, we recommend that non-specialized regional centers emphasize on the importance of rapid transfer to a specialized acute SCI-center before spinal surgical management in order to optimize mechanical ventilation support duration. 


\section{Limitations}

The main limitation of this study is the small number of patients for Group 2. However, we agree that it could be useful, but we were still able to verify our hypothesis and observe a significant influence of the transfer delay on the respiratory management despite the limited number of patients in Group 2. Also, the design of this study may not be the ideal one for answering our important question. As a randomized-control trial is not possible, a larger cohort of patients with prospectively-collected data in which all potential confounders are identified, would have been beneficial. This would have allowed the use of a directed acyclic graph approach [38] yielding an unbiased estimate of the effect, and the subsequent use of a more conceptual point of view.

In addition, although our data acquisition in our specialized acute SCI-center was prospective, it was impossible to collect all the data pertaining to the referring nonspecialized center, such as the initial rate of pneumonia, the duration of MV and the initial neurological status (ISNSCI). As mentioned previously, the rate of pneumonia and duration of MV in Group 2 were likely underestimated because they could not be collected before admission to our specialized acute SCI-center. Indeed, obtaining these data could only have strengthened the conclusions of this study supporting improved respiratory management for Group 1 patients. The ISNCSCI had to be collected at admission to the specialized acute SCI-center for both groups and was thus compared in the pre- and post-operative period for Group 1 and 2 respectively. As only individuals with a complete SCI were included in this study, it is less likely to have influenced results of this study. ISNCSCI (AIS scale) is suggested to be collected also in non-specialized centers and transmitted to the specialized acute SCI-center at the time of transfer.

\section{Conclusions}

Individuals sustaining acute traumatic complete tetraplegia may be first transported to local hospital centers from the trauma site to stabilize the medical condition and establish the diagnosis. Subsequent transfer to a specialized acute SCI-center prior to spinal surgical management decreased the need for tracheostomy placement and reduced MV duration. Weaning from MV support following complete tetraplegia is complex and is very different from other critically ill patients. A specialized team, experienced with the pulmonary physiologic changes specific to complete cervical SCI, should initiate MV weaning in a timely fashion. Admission to a specialized acute SCI-center before surgical management provides a coordinated multidisciplinary approach focusing on the optimization of the respiratory outcomes throughout the different phases of acute care management.

\section{Data archiving}

All relevant data are within this manuscript and raw data are archived by the authors.

Acknowledgements This research was funded by the MENTOR Program of the Canadian Institute of Health Research and by the US Department of Defense Spinal Cord Injury Research Program. Part of the data was collected through the Rick Hansen Spinal Cord Injury Registry.

\section{Compliance with ethical standards}

Conflict of interest The authors declare that they have no competing interests.

\section{References}

1. Zimmer MB, Nantwi K, Goshgarian HG. Effect of spinal cord injury on the respiratory system: basic research and current clinical treatment options. J Spinal Cord Med. 2007;30:319-330.

2. Wong SL, Shem K, Crew J. Specialized respiratory management for acute cervical spinal cord injury: a retrospective analysis. Top Spinal Cord Inj Rehabil. 2012;18:282-290.

3. Thompson C, Much J, Parent S, Mac-Thiong JM. Changing demographics of traumatic spinal cord injury: An 11-year study of 831patients. J Spinal Cord Med. 2015;38:214-23.

4. Kattail D, Furlan JC, Fehlings MG. Epidemiology and clinical outcomes of acute spine trauma and spinal cord injury: experience from a specialized spine trauma in Canada in comparison with a large national registry. $\mathrm{J}$ Trauma. 2009;67:936-43.

5. Kumar N. Respiratory care in traumatic spinal cord injury. J Spine. 2016;S7:1-4.

6. Chasha S, Christie S. Systematic review of intensive cardiopulmonary management after spinal cord injury. J Neurotrauma. 2011;28:1479-95.

7. Brown R, Di Marco AF, Hoit JD, Garshick E. Respiratory function and management in spinal cord injury. Respir Care. 2006;51:853-70.

8. Kornblith LZ, Kutcher ME, Callcut RA, Redick BJ, Hu CK, Cogbill TH, et al. Mechanical ventilation weaning and extubation after spinal cord injury: a western trauma association multicenter study. J Trauma Acute Care Surg. 2013;75:1060-70.

9. Berlowitz DJ, Wadsworth B, Ross J. Respiratory problems and management in people with spinal cord injury. Breathe. 2016;12:328-40.

10. Ganuza JR, Oliviero A. Tracheostomy in spinal cord injured patients. Transi med UniSa. 2011;1:151-72.

11. Charlifue S, Apple D, Burns SP, Chen D, Cuthbert JP, Donovan WH, et al. Mechanical ventiltation, health, and quality of life following spinal cord injury. Arch Phys Med Rehab. 2011;92:457-63.

12. Zakrasek EC, Neilson JL, Kosarchuk JJ, Crew JD, Ferguson AR, McKenna SL. Pulmonary outcomes following specialized respiratory management for acute cervical spinal cord injury: a retrospective analysis. Spinal Cord 2017;55:559-65. 
13. Consortium for Spinal Cord Medicine et al. Early acute management in adults with spinal cord injury: a clinical practice guideline for health-care professionals. J Spinal Cord Med. 2008;31:403-79.

14. Parent S, Barchi S, LeBreton M, Casha S, Fehlings MG. The impact of specialized centers of care for spinal cord injury on length of stay, complications and mortality: a systematic review of the literature. J Neurotrauma. 2011;28:1363-70.

15. Consortium for Spinal Cord Medicine et al. Respiratory management following spinal cord injury: a clinical practice guideline for health-care providers. J Spinal Cord Med. 2005;28: 259-93.

16. Stevens RD, Bhardwaj A, Kirsh JR, Mirski MA. Critical care and management in traumatic spinal cord injury. J Neurosurg Anesth. 2003;15:215-29.

17. Mac-thiong JM, Feldman D, Thompson C, Bourassa-Moreau E, Parent S. Does timing of surgery affect hospitalization costs and length of stay for acute care following a traumatic spinal cord injury? J Neurotrauma. 2012;29:2816-22.

18. Bourassa-Moreau E, Mac-thiong JM, Feldman DE, Thompson C, Parent S. Non-neurological outcomes after complete traumatic spinal cord injury: the impact of surgical timing. J Neurotrauma. 2013;30:1596-601.

19. Bourassa-Moreau E, Mac-thiong JM, Li A, Erhmann Feldman D, Gagnon DH, Parent S. Do patients with complete spinal cord injury benefit from early surgical decompression? Analysis of neurological improvement in a prospective cohort study. J Neurotrauma. 2016;33:301-6.

20. McKinley W, Meade MA, Kirshblum S, Barnard B. Outcomes of early surgical management versus late or no surgical intervention after acute spinal cord injury. Arch Phys Med Rehab. 2004;85:1818-25.

21. Richard-Denis A, Ehrmann Feldman D, Thompson C, Bourassa-Moreau E, Mac-Thiong JM. Costs and length of stay for the acute care of patients with motor-complete spinal cord injury following cervical trauma: the impact of early transfer to specialized acute SCI center. Am J Phys Med Rehabil. 2017;96:449-456.

22. Richard-Denis A, Feldman ED, Thompson C, Mac-Thiong JM. The impact of acute management on the occurrence of medical complications during the specialized spinal cord injury acute hospitalization following motor-complete cervical spinal cord injury. J Spinal Cord Med. 2017;19:1-18.

23. Tuski DS. The impacts of the model SCI system: historical perspective. J Spinal Cord Med. 2002;25:301-5.

24. Quershi AZ. Tracheostomy decannulation; A cath-22 for patients with spinal cord injuries. Int J Phys Med Rehabil. 2013;1:1-3.

25. Kirshblum SC, Burns SP, Biering-Sorensen F, Donovan W, Graves DE, Amitabh $\mathrm{J}$, et al. International standards for neurological classification of spinal cord injury (Revised 2011). J Spinal Cord Med. 2011;34:535-546.

26. Furlan JC, Tung K, Fehlings MG. Process benchmarking appraisal of surgical decompression of spinal cord following traumatic cervical spinal cord injury: opportunities to reduce delays in surgical management. J Neurotrauma. 2013;30:487-491.

27. Gupta B, Agrawal P, D'souza N, Dev Soni K. Start time delays in operating room: different perspectives. Saudi J Anesth. 2011;5:286-8.

28. Fehlings MG, Vaccaro A, Wilson JR, Singh A, Cadotte WD, Harrop JS, et al. Early versus delayed decompression for traumatic cervical spinal cord injury: results of the surgical timing in acute spinal cord injury study (STASCIS). PLoS ONE. 2012;7:e32037.

29. Roquilly A, Seguin P, Mimoz O, Feuillet F, Rosenczweig E, Chevalier $\mathrm{F}$, et al. Risk factors for prolonged duration of mechanical ventilation in acute traumatic tetraplegic patients-a restrospective cohort study. J Crit Care. 2014;29:313e7-13.

30. Krueger H, Noonan VK, Trenaman LM, Joshi P, Rivers CS. The economic burden of traumatic spinal cord injury in Canada. Chronic Dis Inj Can. 2013;33:113-22.

31. Liu J-M, Long X-H, Zhou Y, Peng H-W, Liu Z-L, Huang S-H. Is urgent decompression superior to delayed surgery for traumatic spinal cord injury? A meta-analysis. World Neurosurg. 2016;87:124-131.

32. Thompson C, Feldman DE, Mac-Thiong J-M. Surgical management of patients following traumatic spinal cord injury: identifying barriers to early surgery in a specialized spinal cord injury center. J Spinal Cord Med 2016;8:1-7.

33. Berlly M, Kazuko S. Respiratory management during the first five days after spinal cord injury. J Spinal Cord Med. 2007;30:309-18.

34. Nogueira Nemer S, Valente Barbas CS. Predictive parameters for weaning from mechanical ventilation. J Bras Pneumol. 2011;37:669-79.

35. Como JJ, Sutton ERH, McCunn M, Dutton RP, Johnson SB, Arabi $\mathrm{B}$, et al. Characterizing the need for mechanical ventilation following cervical spinal cord injury with neurologic deficits. J Trauma. 2005;59:912-5.

36. Fishburn MJ, Marino RJ, Ditunno JF Jr. Atelectasis and pneumonia in acute spinal cord injury. Arch Phys Med Rehab. 1990;71:197-200.

37. Macciocchi S, Steel RT, Warshowsky A, Thompson N, Barlow K. Co-occurring traumatic brain injury and acute spinal cord injury rehabilitation outcomes. Arch Phys Med Rehab. 2012;93:1788-94.

38. Shrier I, Platt RW. Reducing bias through directed acyclic graphs. BMC Med Res Methodol. 2008;8:70. 